\title{
Special issue on advanced intelligent multimedia applications for next generation environments
}

\author{
Jong Hyuk Park • Hamid R. Arabnia • Zhiwen Yu
}

Published online: 2 December 2009

(C) Springer Science+Business Media, LLC 2009

Next generation (NG) environments have recently raised relevant interests in both the academic and industrial research communities as one of the most promising application fields for intelligent computing services. In fact, the NG application domain poses unique challenges such as various services to enable working from home, remote education, remote diagnosis, virtual shopping, network gaming, and portal \& high quality VOD with no limitations on space and time, and so on. Specially, advanced intelligent multimedia applications for NG have been coming as a new paradigm. This special issue aims to address the advanced intelligent multimedia applications for NG.

Submissions to this special issue come from an open call for papers as well as from selected papers presented at the 2008 International Symposium on Ubiquitous Multimedia Computing (UMC-08) held at Hobart, Australia, from 13 to 15 October, 2008. Accordingly, a number of papers included in this special issue concentrate on advanced intelligent multimedia applications including topics like smart homes, intelligent multimedia systems, human communication interaction, context-awareness, advanced image processing, security issues that are associated with next generation multimedia applications.

Kim et al. has proposed a shape-based index matching scheme for camera view invariant 3-D object retrieval. Especially, in order to remove redundant camera views to be indexed, authors investigate the symmetrical shape pattern of objects and propose two camera view skimming methods, which are mirror shape pairing and camera view pruning. Through

\footnotetext{
J. H. Park $(\bowtie)$

Department of Computer Science and Engineering, Seoul National University of Technology, Seoul, South Korea

e-mail: parkjonghyuk1@hotmail.com

H. R. Arabnia

Department of Computer Science, University of Georgia, Athens, GA, USA

e-mail: hra@cs.uga.edu

Z. $\mathrm{Yu}$

School of Computer Science, Northwestern Polytechnical University, Xi'an, Shaanxi,

People's Republic of China

e-mail: zhiwenyu@nwpu.edu.cn
} 
various experiments, authors show that their proposed scheme can achieve excellent performance.

Jui-Hung Chang, et al. proposed a mechanism which uses datamining techniques such as k-means and k-nearest-neighbors in P2P social network, to cluster users by their TV channel watching history and watching behavior (Personal Behavior Profile). After users are classified, popular programs can be recommended to each cluster of users specifically. A more convenient mean is provided to users for selecting TV program.

M. Tsai, et al. propose a sub-packet level forward error correction mechanism that can find an appropriate number of sub-packets in accordance with a mathematical model to minimize the checksum and FEC redundancy overhead. The proposed mechanism outperforms the traditional byte-level and packet-level forward error correction mechanisms in term of effective packet loss rate, recovery overhead, and peak signal to noise ratio.

To facilitate the development and proliferation of context-aware e-learning services, Zhiwen $\mathrm{Yu}$, et al. propose an e-learning infrastructure called Semantic Learning Space. The software infrastructure leverages the Semantic Web technologies to support semantic knowledge representation, systematic context management, interoperable content integration, expressive knowledge query, and adaptive content recommendation. The functionality encapsulated in the infrastructure handles the common, time-consuming and low-level details in learning context processing and content management.

Ibrahim Kamel et al. propose an algorithm for bandwidth management at the residential gateway $(\mathrm{RG})$. The proposed algorithm control the amount of non-real-time TCP traffic on the broadband access link from the Internet Service Provider (ISP) to the RG so that the bandwidth requirements of the real-time traffic are satisfied. The basic idea is to dynamically manipulate the flow control window sizes based on the total available bandwidth for the non-real-time traffic.

Gerald Schaefer in his contribution focusses on the problem of effectively managing large image collections. Image thumbnails are projected on a spherical visualisation body which represents the user's browsing environment. Images are organised in a hierarchical way allowing the user to intuitively navigate through the visualisation structure. The proposed system is shown to represent a true alternative to common query-based retrieval approaches.

Misra and Mohanta propose a protocol for energy-efficient adaptive listen for medium access control in Wireless Sensor Networks (WSN). Their proposed protocol adaptively changes the slot-time, which is the time of each slot in the contention window. This correspondingly changes the cycle-time, while keeping the duty-cycle constant. Using simulation experiments, the authors show that the performance of their protocol is better than the popular SMAC protocol.

Soo-Kyun Kim, et al. proposes a generation scheme of a simplified model for real-time human face animation in intelligent multimedia systems. The quality of facial animation with a lower-level model is as good as that of a computationally expansive original model. In this paper, authors prove that our decimated facial model is effective in facial animation using a well-known expression-retargeting technique.

Yun-Feng Chou and Zen-Chung Shih propose a statistical model based on regression analysis. Kernel regression with elliptic radial basis functions is used to represent the shape of a character and animate the character with an expressive face. Locally weighted regression is applied to preserve the details within the animated character. The results show that their model synthesizes natural movements of a character and allows virtual humans generation. 
In "Wireless Multimedia Delivery over 802.11e with Cross-layer Optimization Techniques", Chilamkurti et al. propose a cross-layer mapping algorithm aimed at improving the quality of H.264 video over IEEE 802.11e-based networks. By mapping relevant H.264 video slices to appropriate $802.11 \mathrm{e}$ access categories, the authors demonstrate better video performance (in terms of PSNR, frame loss, etc) with their proposed cross-layer approach over the traditional EDCA scheme.

Ivan Lee and Jong Hyuk Park investigate frame loss rate of distributed video streaming over multiple independent-paths. Multiple description coding is adopted to provide scalability over different network conditions, and the impact of drift error due to lossy data transmission is simulated using the Gilbert model. An adaptive scheme for adjusting group-of-pictures of the multiple description video codec is studied, and the simulation results demonstrate an improvement in the reconstructed video quality

Finally, we strongly believe that the selected papers make a significant contribution to researchers, practitioners and students working in the areas of the advanced intelligent multimedia applications for NG. We would like to express our sincere appreciation to all the authors for their valuable contributions and also to the referees for their cooperation and hard work in reviewing the papers in a timely and professional manner. Our special thanks go to the guest editorial board for this SI and professor Borko Furht who is the Editor-inChief of Multimedia Tools and Applications for his support throughout the whole publication processes.

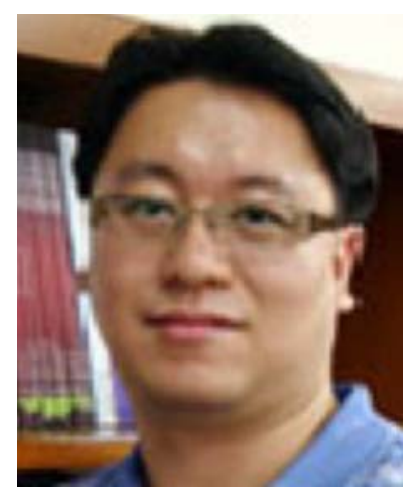

Jong Hyuk Park received his Ph.D. degree in Graduate School of Information Security from Korea University, Korea. From December, 2002 to July, 2007, Dr. Park had been a research scientist of R\&D Institute, Hanwha S\&C Co., Ltd., Korea. From September, 2007 to August, 2009, He had been a professor at the Department of Computer Science and Engineering, Kyungnam University, Korea. He is now a professor at the Department of Computer Science and Engineering, Seoul National University of Technology, Korea. Dr. Park has published about 100 research papers in international journals and conferences. He has been serving as chairs, program committee, or organizing committee chair for many international conferences and workshops. He was editor-in-chief of the International Journal of Multimedia and Ubiquitous Engineering (IJMUE), the managing editor of the International Journal of Smart Home (IJSH). He is Associate Editor / Editor of 14 international journals including 8 journals indexed by SCI(E). In addition, he has been serving as a Guest Editor for international journals by some publishers: Springer, Elsevier, John Wiley, Oxford Univ. press, Hindawi, Emerald, Inderscience. His research interests include security and digital forensics, ubiquitous and pervasive computing, context awareness, multimedia services, etc. He got the best paper award in ISA-08 conference, April, 2008. And he got the outstanding leadership awards from IEEE HPCC09 and ISA-09, June, 2009. 


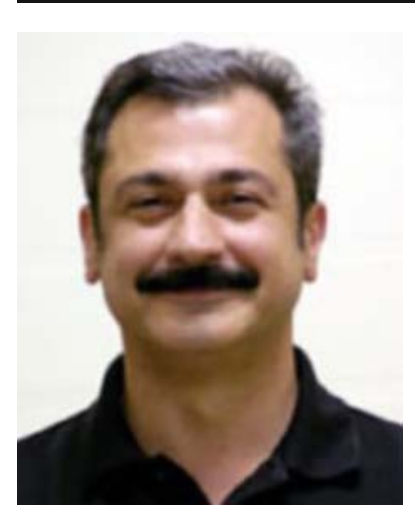

Hamid R. Arabnia received a Ph.D. degree in Computer Science from the University of Kent (Canterbury, England) in 1987. Arabnia is currently a Full Professor of Computer Science at University of Georgia (Georgia, USA), where he has been since October 1987. His research interests include Parallel and distributed processing techniques and algorithms, interconnection networks, and applications (in particular, in image processing, medical imaging, and other computational intensive problems). Arabnia is Editor-in-Chief of The Journal of Supercomputing published by Springer (https:/www.editorialmanager.com/supe/) and is on the editorial and advisory boards of 26 other journals and magazines. Arabnia has received numerous number of awards, including, The Johns Hopkins University National Search (Certificate of Achievement) in recognition of his contributions to the national program for enhancing the quality of life for people with disabilities through the application of computing technology (presented to him in December 1991signatories: co-directors of the National Search and President of Johns Hopkins U). More recently (October 14, 2007), he received an "Outstanding Achievement Award in Recognition of His Leadership and Outstanding Research Contributions to the Field of Supercomputing". This award was formally presented to him at Harvard University Medical School (signatories: Lawrence O. Hall, President of IEEE/SMC; Zhi-Pei Liang, Vice President of IEEE/EMB; Jack. Y. Yang, General Chair of IEEE BIBE and Harvard University; Mary Qu Yang, Chair of Steering Committee, IEEE BIBE and NIH). Arabnia has published extensively in journals and refereed conference proceedings. He has over 300 publications (journals, proceedings, editorship, editorials) in his areas of research. Arabnia has delivered numerous number of keynote lectures at international conferences; most recently at (since September 2008): The 14th IEEE International Conference on Parallel and Distributed Systems (ICPADS'08, Australia); International Conference on Future Generation Communication and Networking (FGCN 2008 / IEEE CS, Sanya/China); The 10th IEEE International Conference on High Performance Computing and Communications (HPCC-08, Dalian/China), ... He has also delivered a number of "distinguished lectures" at various universities. 


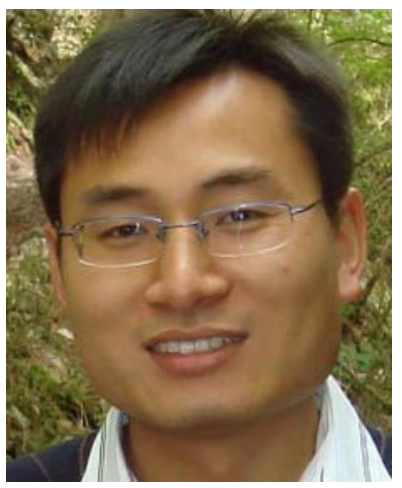

Dr. Zhiwen Yu is currently a professor at the School of Computer Science, Northwestern Polytechnical University, P. R. China. He received his B.Eng, M.Eng and Ph.D degree of Engineering in computer science and technology in 2000, 2003 and 2005 respectively from the Northwestern Polytechnical University. He has worked as a research fellow at Kyoto University, Japan from Feb. 2007 to Jan. 2009, and a post-doctoral researcher at Nagoya University, Japan in 2006-2007. He has been a visiting researcher at Institute for Infocomm Research, Singapore from Sep. 2004 to May 2005. He is the Associate Editor of the International Journal of Multimedia and Ubiquitous Engineering (IJMUE) and International Journal of Technology Enhanced Learning (IJTEL). He serves as the guest editor of ACM Multimedia Systems Journal, Personal and Ubiquitous Computing (Springer), Pervasive and Mobile Computing (Elsevier), and Cybernetics and Systems (Taylor \& Francis). He is the Program Chair of UIC 2010, Co-Organizer of PMPC 2009, Program Chair of SensorFusion'07/09 and SmarTel'07/09. He serves as the Publicity Chair of PerCom 2010 and PERVASIVE 2008. He has also served as PC member for a number of conferences, e.g., IEEE PerCom, DEXA, EuroITV, MMSP, KES, ACM SAC, IEEE AINA, EUC, ICOST, GPC, ADCOM, PerEL, ICESS, MUE, MoMM, CARPE, IE, etc. Dr. Yu has published around 70 scientific papers in refereed journals and conferences, e.g., ACM Computing Surveys, IEEE Pervasive Computing, IUI, etc. His research interests cover pervasive computing, context-aware systems, human-computer interaction, and personalization. Zhiwen $\mathrm{Yu}$ is a member of the IEEE and IEEE Computer Society, CCF, and CCF Pervasive Computing Technical Committee. He received a Humboldt Fellowship in 2008, the UIC 2009 Best Paper Award, and the Excellent Doctoral Dissertation Award from China Computer Federation in 2006. 